\title{
Ectopic Tubular Papillary Apocrine Adenoma on the Eyelid: A Case Report of Misleading Clinical Presentation and Location
}

\author{
Laura Abbeel Rita Van Ginderdeuren \\ Department of Ophthalmology and Pathology, University Hospital Leuven, Leuven, Belgium
}

\section{Established Facts}

- Tubular papillary apocrine adenoma is a diagnostically challenging, clinical, and pathological entity, most commonly reported on the scalp.

\section{Novel Insights}

- Pathological examination is important to define the apocrine origin and exclude malignancy, when clinical features and ectopic location may complicate diagnosis.

\section{Keywords}

Eyelid · Tubular papillary apocrine adenoma · Moll gland . Apocrine sweat gland

\begin{abstract}
We report the case of a 38-year-old patient with a tubular apocrine adenoma with papillary differentiation. The clinician was challenged in her diagnosis by the initially misleading appearance and ectopic location. Histopathological findings and immunohistochemistry delivered conclusive proof for the correct diagnosis. Therefore, it is important for the ophthalmologist to request histopathological analysis to diagnose and differentiate from malignancy. A complete local excision is recommended to prevent recurrence.
\end{abstract}

(c) 2018 S. Karger AG, Basel

\section{KARGER}

(c) 2018 S. Karger AG, Basel

E-Mail karger@karger.com

www.karger.com/oop

\section{Introduction}

Sweat glands in the eyelid are classified as being either apocrine or eccrine in origin. Histopathologically, the apocrine features are manifested by deeply eosinophilic, columnar-to-cuboidal epithelium with basal nuclei and apical cytoplasmic snouts of decapitation secretion. Water-producing cysts lined by low, cuboidal, or flattened epithelium are classified as eccrine. The former tends to be much more common in the eyelids than their eccrine counterparts [1]. These apocrine glands were initially reported by - and named after the Dutch ophthalmologist - Jacob Moll (1832-1914), and Moll gland neoplasms are still considered to be clinically and pathologically a diagnostic challenge. A tubular apocrine adenoma (TAA) or tubular papillary adenoma (TPA) with apocrine dif- 

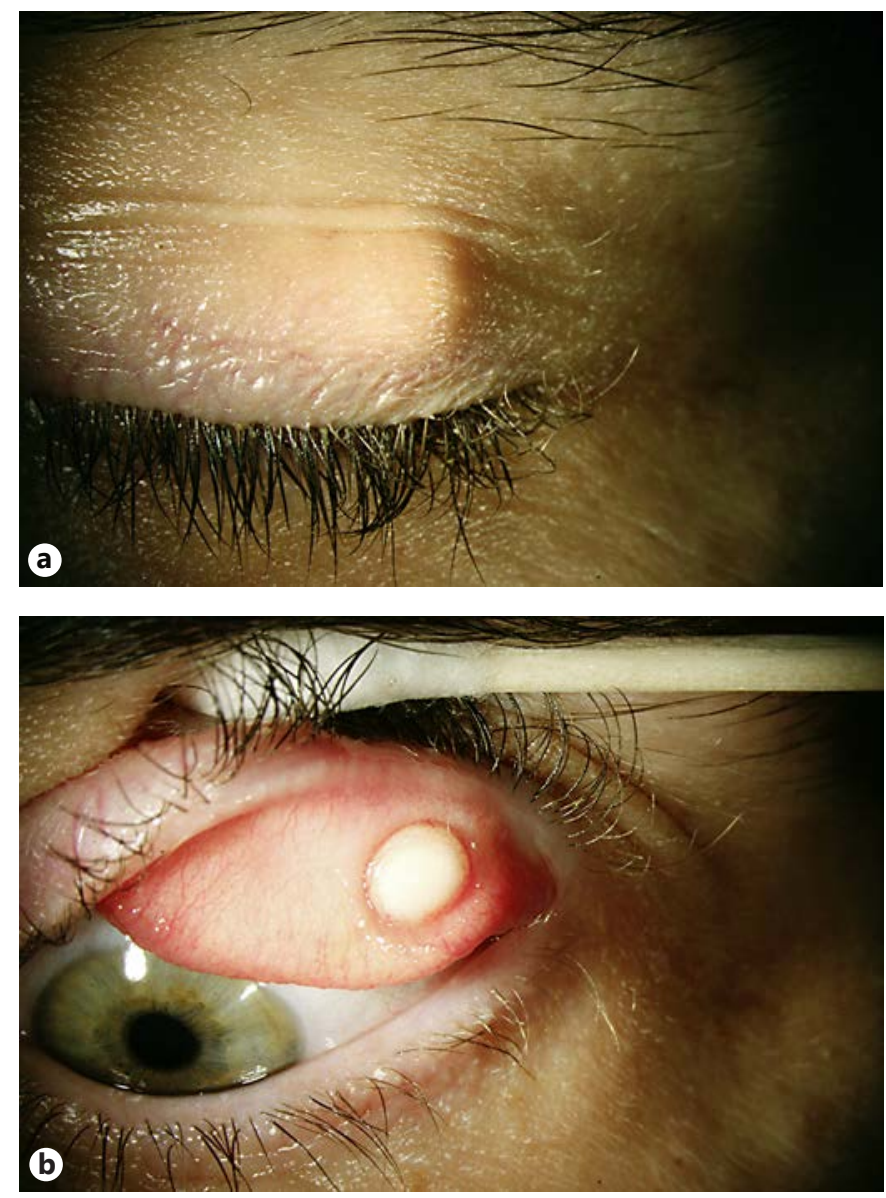

Fig. 1. Clinical appearance before surgery. a Solid mass in the middle of the tarsus of the left upper eyelid. b Solid white-yellow lesion, no local inflammation.

ferentiation describes a rare and benign sweat gland tumor, most commonly to be found as a nodular solitary lesion on the scalp and first described by Landry and Winkelmann in 1972 [2]. It is described to contribute to a common spectrum of different apocrine neoplasms with distinct architectural characteristics. In this context, we report a TPA with apocrine differentiation found in an ectopic location of the conjunctival tarsal side of the upper eyelid.

\section{Case Report}

A 38-year-old woman presented with a 12-month history of an upper eyelid lesion in the middle of her left eye (Fig. 1a). There was no previous ophthalmic history of the left eye; however, she had undergone strabismus surgery on the right, divergent, amblyopic eye at the age of 3 years. She denied any pain or rapidly expanding growth of the lesion in the given period. A hard, solid mass could be felt during palpation of the skin. When everting the eyelid, we found a well-circumscribed, solid, yellow mass that seemed to be located posterior to the tarsus, covered by conjunctiva (Fig. 1b). Further ocular and orbital examination was unremarkable. The clinician's preoperative impression was that the lesion represented a tarsal plate cyst or an atypical chalazion without local redness due to inflammation. Whilst performing the excision, the tumor was found to be located entirely at the conjunctival side of the tarsus.

The eyelid was everted, and the lesion was excised en bloc together with the posterior side of the tarsus, leaving the bare tarsal plate. At the end of the surgery, this resulted in an intact eyelid margin of $3 \mathrm{~mm}$. The wound was left to heal without sutures, with the application of dexamethason-tobramycin ointment for 10 days and a temporary eye pad. The solid tumor was conserved in toto in $10 \%$ buffered formalin and processed to paraffin sections. Fourmm-thick sections were cut and stained with hematoxylin and eo$\sin (\mathrm{H} \& \mathrm{E})$ or periodic acid-Schiff (PAS).

Histopathological examination revealed an eyelid biopsy with part of the fibrous tarsal plate included. On the conjunctival side, a sessile, flattened, papillary tumor was found consisting of multiple interconnecting tubules in a thin fibrous stroma. The tumor's origin was defined at the conjunctival posterior side of the tarsus without invasion through the fibrous plate into the anterior lamella (Fig. 2a). The tubules were all lined with a continuous bilayer epithelium of large columnar cells on the luminal side and smaller cuboidal or flattened cells on the basal side. The luminal cells showed a homogeneous, eosinophilic cytoplasm and PASpositive decapitation snouts on the PAS and H\&E staining (Fig. 2a-d).

All nuclei were bland, round to oval, rarely with a small nucleolus and without any signs of atypia or pleiomorphism. No mitoses could be detected. The underlying stroma contained few small vessels and some lymphocytes but very few plasma cells. Immunostaining with cytokeratin 7 (Fig. 3) and epithelial membrane antigen was positive in the 2 layers of the tubules. Alpha-smooth muscle actin and androgen receptor were negative. CD138 showed only very few plasma cells in the stroma. Ki-67 was completely negative in the epithelial layers. Alcian blue staining was not performed.

\section{Discussion}

In this report, we describe a very rare benign eyelid TAA at an ectopic location. Even though multiple apocrine glands (or glands of Moll) are present in the eyelid, very few benign tumors with apocrine origin have been published at this location, besides hidrocystomas which are found along the eyelid margin and canthi. Following the WHO classification of apocrine tumors, the terms apocrine adenoma, tubular adenoma, tubulopapillary hidradenoma, and papillary tubular adenoma are synonyms [3].

The TPA with apocrine differentiation (also called tubular apocrine adenoma [TAA]) is described as part of a 

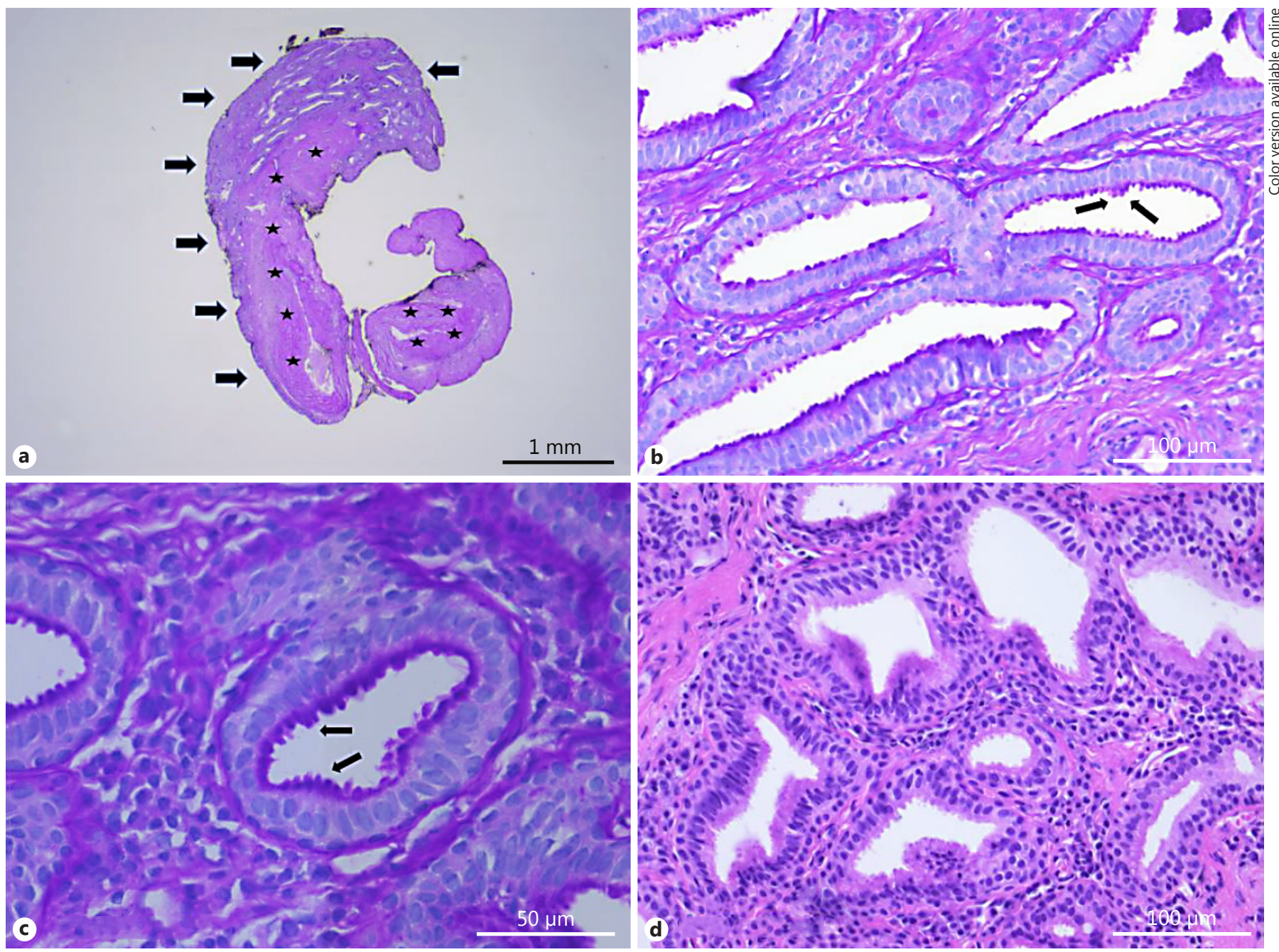

Fig. 2. a Microscopic picture: overview of the lesion. The tumor is on the left (indicated by the arrows). The tarsal plate is on the right (indicated by stars). PAS staining (magnification $\times 1.6$, scale bar $=$ $1 \mathrm{~mm}$ ). b Microscopic picture: apical decapitation snouts, typical for an apocrine origin (arrows). PAS staining (magnification $\times 20$,

common spectrum of 3 apocrine neoplasms. TPA with apocrine differentiation, syringocystadenoma papilliferum (SCAP), and apocrine gland cyst (AGC, also called apocrine hidrocystoma or apocrine cystadenoma) are reported to represent one spectrum with distinct architectural characteristics [4]. This idea has been supported by cases being published with either a varying degree of or simultaneous presence of these different entities in 1 lesion, which was initially described by Toribio et al. [5] in 1987 [6]. SCAP is composed of cysts and consists of a double layer of epithelial cells. The stroma often shows rich infiltration of inflammatory plasma cells,

scale bar $=100 \mu \mathrm{m})$. c Details of apocrine origin: apical decapitation snouts (arrows). PAS staining (magnification $\times 40$, scale bar $=$ $50 \mu \mathrm{m})$. d Microscopic picture: cellular and nuclear details of the columnar, benign tumor cells. $\mathrm{H} \& \mathrm{E}$ staining (magnification $\times 20$, scale bar $=100 \mu \mathrm{m})$.

which is rarely detected in TPA and AGC. AGC has unilocular or multilocular cysts. TPA has been described as a nodular mass composed of multiple tubules outlined by a dual layer of epithelium with an apocrine type or decapitation secretion within the luminal cell layer. TPA differs in architecture from SCAP by (a) the lack of papillary projections, (b) no cystic invaginations extending down from the epidermis, and (c) the absence of inflammatory plasma cells [4].

The apocrine origin of this lesion was proven by histopathological features and immunohistochemistry. The cardinal feature of TPA with apocrine differentiation in- 


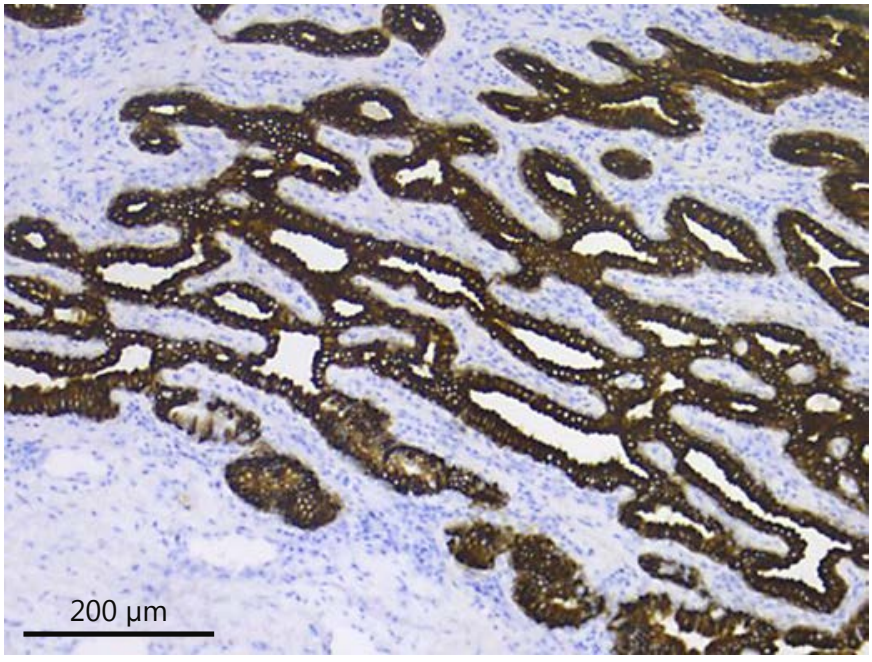

Fig. 3. Immunohistochemistry. Cytokeratin 7 staining (magnification $\times 10$, scale bar $=200 \mu \mathrm{m})$.

cludes having a double layer of inner columnar and outer cuboidal cells and the presence of apical decapitation secretion in the inner columnar cells rather than forming simple secretory units with tubules, which is the case with their eccrine counterpart [7]. In the surrounding stroma consisting of collagen and fibroblast, very little plasma cell infiltration was present, detected with CD138 antibody staining. Malignancy was excluded due to a normal organization in structure and the absence of mitotic figures and atypia and no elevated Ki-67 staining.

Of the 2 types of sweat glands in the eyelids, only the apocrine glands of Moll are described as exclusively localized at or near the eyelid margin and canthi. Eccrine sweat glands, however, are not found at the eyelid margin but are found several millimeters from the lid margin in pretarsal and preseptal skin $[1,8]$. In our patient, the location on the conjunctival tarsal side of the upper eyelid is rather unusual for its apocrine origin and would not raise obvious clinical suspicion, even though apocrine glands are found in the near proximity of the margin.

A TAA typically presents in the scalp region but has also been reported on the eyelid in previous cases [9-12]. However, these reports described a TAA with an intradermal location on the eyelid, whereas the specimen in our case has its origin at the posterior conjunctival border of the tarsus. Hence, to our knowledge, our case could be considered as the first report of an ectopic TPA with apocrine differentiation in the eyelid.

When considering the differential diagnosis in our patient, the initial assessment of a solid lesion at the mid- tarsus with a yellow color on eversion of the eyelid would not raise suspicion for a TAA. Instead, one could strongly suspect a more common, intratarsal, keratinous cyst of the eyelid, due to its clinical appearance, the fixation of the lesion onto the tarsus, and the subconjunctival location [13].

In conclusion, a TPA with apocrine differentiation on the eyelid is a rare and diagnostically challenging benign entity. This case is, to our knowledge, the first report in the English literature of an ectopic presentation in the eyelid and demonstrates that the appearance and location could be misleading in diagnosing this benign apocrine sweat gland tumor. We, therefore, strongly recommend relying on histopathological analysis, to demonstrate characteristic features such as apical decapitation snouts, the limited presence of plasma cells, and immunohistochemical staining as a conclusive guidance. Furthermore, a complete local excision is required to prevent recurrence.

\section{Statement of Ethics}

The authors declare that the undertaken research complies with the guidelines for human studies. The subject has given informed consent; no study protocol was required for this case report.

\section{Disclosure Statement}

The authors declare that there are no financial interests or conflicts of interest related to this paper.

\footnotetext{
References $\quad D_{1}$ Jakobiec FA, Zakka FR: A reappraisal of eyelid eccrine and apocrine hidrocystomas: microanatomic and immunohistochemical studies of 40 lesions. Am J Ophthalmol 2011;151: 358-374.

2 Landry M, Winkelmann RK: An unusual tubular apocrine adenoma. Arch Derm 1972; 105:869-879.

3 LeBoit PE, Burg G, Weedon D Sarasain A (eds.): World Health Organization Classification of Tumours; Pathology and Genetics of Skin Tumours. Lyon, IARC Press, 2006, pp 145-146.

4 Ansai SI, Anan T, Fukumoto T, Saeki H: Tubulopapillary cystic adenoma with apocrine differentiation: a unifying concept for syringocystadenoma papilliferum, apocrine gland cyst, and tubular papillary adenoma. Am J Dermatopathol 2016;0:1-9.
} 
5 Toribio J, Zulaica A, Peteiro C: Tubular apocrine adenoma. J Cutan Pathol 1987;14:114117.

-6 Lee HJ, Cho E, Kim MH, Cho SH, Lee JD: Syringocystadenoma papilliferum of the back combined with a tubular apocrine adenoma. Ann Dermatol 2011;23(suppl 2):S151-S154.

$\checkmark 7$ Obaidat NA, Alsaad KO, Ghazarian D: Skin adnexal neoplasms - part 2: an approach to tumours of cutaneous sweat gland. J Clin Pathol 2007;60:145-159.
-8 Jakobiec FA, Rai R, Lefebvre DR: Papillary hidradenoma of the eyelid margin: clinical and immunohistochemical observations further supporting an apocrine rather than an eccrine origin. Surv Ophthalmol 2014;59:540-547.

9 Stokes J, Ironside J, Smith C, Dhillon D: Tubular apocrine adenoma - an unusual eyelid tumour. Eye 2005;19:237-239.

10 Raven ML, Krivochenitser RI, Lucarelli MJ: Tubular apocrine adenoma in association with an apocrine hidrocystoma. Ophthalmology 2016;123:2385.

-11 Barker Griffith AE, Streeten BW, Charles NC: Moll gland neoplasms in the eyelid: a clinical and pathological spectrum in 5 cases. Arch Ophthalmol 2006;124:1645-1649.
12 Ersanli A, Turan-Vural E, Comunoglu C, Ercalik Y: Multiple tubular adenoma apocrine on upper and lower eyelid. Int J Ophthalmic Pathol 2014;3:3.

13 Jakobiec FA, Mehta M, Iwamoto M, Hatton MP, Thakker M, Fay A: Intratarsal keratinous cysts of the Meibomian gland: clinicopathologic and immunohistochemical features in 6 cases. Am J Ophthalmol 2010;149:82-94. 